\title{
A Parametric Finite-Element Model for Evaluating Segmented Mirrors with Discrete, Edgewise Connectivity
}

\author{
Jessica A. Gersh-Range ${ }^{\mathrm{a}}$, William R. Arnold ${ }^{\mathrm{b}}$, Mason A. Peck ${ }^{\mathrm{a}}$, H. Philip Stahl ${ }^{\mathrm{c}}$

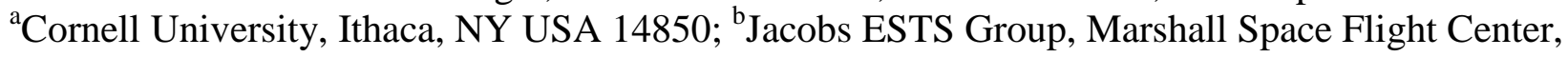 \\ Huntsville, AL USA 35812; NASA, Marshall Space Flight Center, Huntsville, AL USA 35812
}

\begin{abstract}
Since future astrophysics missions require space telescopes with apertures of at least 10 meters, there is a need for on-orbit assembly methods that decouple the size of the primary mirror from the choice of launch vehicle. One option is to connect the segments edgewise using mechanisms analogous to damped springs. To evaluate the feasibility of this approach, a parametric ANSYS model that calculates the mode shapes, natural frequencies, and disturbance response of such a mirror, as well as of the equivalent monolithic mirror, has been developed. This model constructs a mirror using rings of hexagonal segments that are either connected continuously along the edges (to form a monolith) or at discrete locations corresponding to the mechanism locations (to form a segmented mirror). As an example, this paper presents the case of a mirror whose segments are connected edgewise by mechanisms analogous to a set of four collocated singledegree-of-freedom damped springs. The results of a set of parameter studies suggest that such mechanisms can be used to create a 15-m segmented mirror that behaves similarly to a monolith, although fully predicting the segmented mirror performance would require incorporating measured mechanism properties into the model.
\end{abstract}

Keywords: segmented mirror, edgewise connectivity, space telescope

\section{INTRODUCTION}

As future astrophysics missions require space telescopes with apertures of at least 10 meters, there is a need for onorbit assembly methods that decouple the size of the primary mirror from the choice of launch vehicle. For example, answering questions about early star formation, planetary evolution, and the presence of large organic molecules in protostellar disks will require the construction of a 10- to 16-m class far-infrared to submillimeter space telescope; atmospheric extinction precludes ground-based measurements at these wavelengths, and existing space telescopes that operate in this range suffer from source confusion. ${ }^{1-4}$ Similarly, the high-contrast spectroscopy required for searching for biomarkers in the atmospheres of terrestrial-mass exoplanets in the habitable zones of nearby stars is not feasible with even a $30-\mathrm{m}$ ground telescope but well within the capabilities of a $10-\mathrm{m}$ space telescope. ${ }^{2,5}$ Although a monolithic primary may be optically ideal for these space telescopes, such a primary would be too large to fit in any current launch vehicle fairing.

While scaling the design of the James Webb Space Telescope (JWST) ${ }^{6}$ may provide a means of packaging and deploying large segmented primaries, the maximum primary mirror diameter is still limited by the fairing constraints. To enable even larger segmented primaries, a variety of on-orbit assembly methods have been proposed, including formation flight, ${ }^{7}$ electromagnetic formation flight, ${ }^{8}$ and laser trapping. ${ }^{9,10}$ These methods treat the primary mirror as an array of free-floating segments and rely on active control to prevent collisions and maintain optical alignment. An alternative approach is to connect the segments edgewise using mechanisms analogous to damped springs. ${ }^{11}$ In this approach, the assembly procedure consists of multiple stages. It is assumed that there exists some method of maintaining the alignment while the segments are being connected edgewise. Once the segments are connected, the spring-like mechanisms take over the function of maintaining the alignment and phasing. Depending upon the mechanism parameters, it may be possible to form a passively stable segmented primary mirror that behaves similarly to an equivalently shaped monolith.

To evaluate the feasibility of this approach, a parametric ANSYS model that calculates the mode shapes, natural frequencies, and disturbance response of such a mirror, as well as of the equivalent monolithic mirror, has been developed. This model constructs a mirror using rings of hexagonal segments that are either connected continuously along the edges (to form a monolith) or at discrete locations corresponding to the mechanism locations (to form a 
segmented mirror). The details of the model construction and assumptions are presented in Section 2. As an example, this paper presents the case of a mirror whose segments are connected edgewise by mechanisms analogous to a set of four collocated single-degree-of-freedom damped springs, such as flux-pinning mechanisms. ${ }^{11}$ A series of parameter studies is presented in Section 3 and discussed in Section 4, with conclusions offered in Section 5.

\section{MODEL CONSTRUCTION AND CAPABILITIES}

\subsection{Model geometry, connectivity, and assumptions}

To investigate the behavior of a mirror whose segments are connected edgewise by mechanisms analogous to damped springs, a parametric ANSYS model has been developed. This model constructs a mirror using hexagonal segments and a set of geometric parameters that includes the mirror diameter, the size of the gap between the segments, the number of rings, the mirror curvature, and the aspect ratio. The choice of a segmented mirror or its equivalent monolith determines the segment connectivity, as shown in Figure 1.

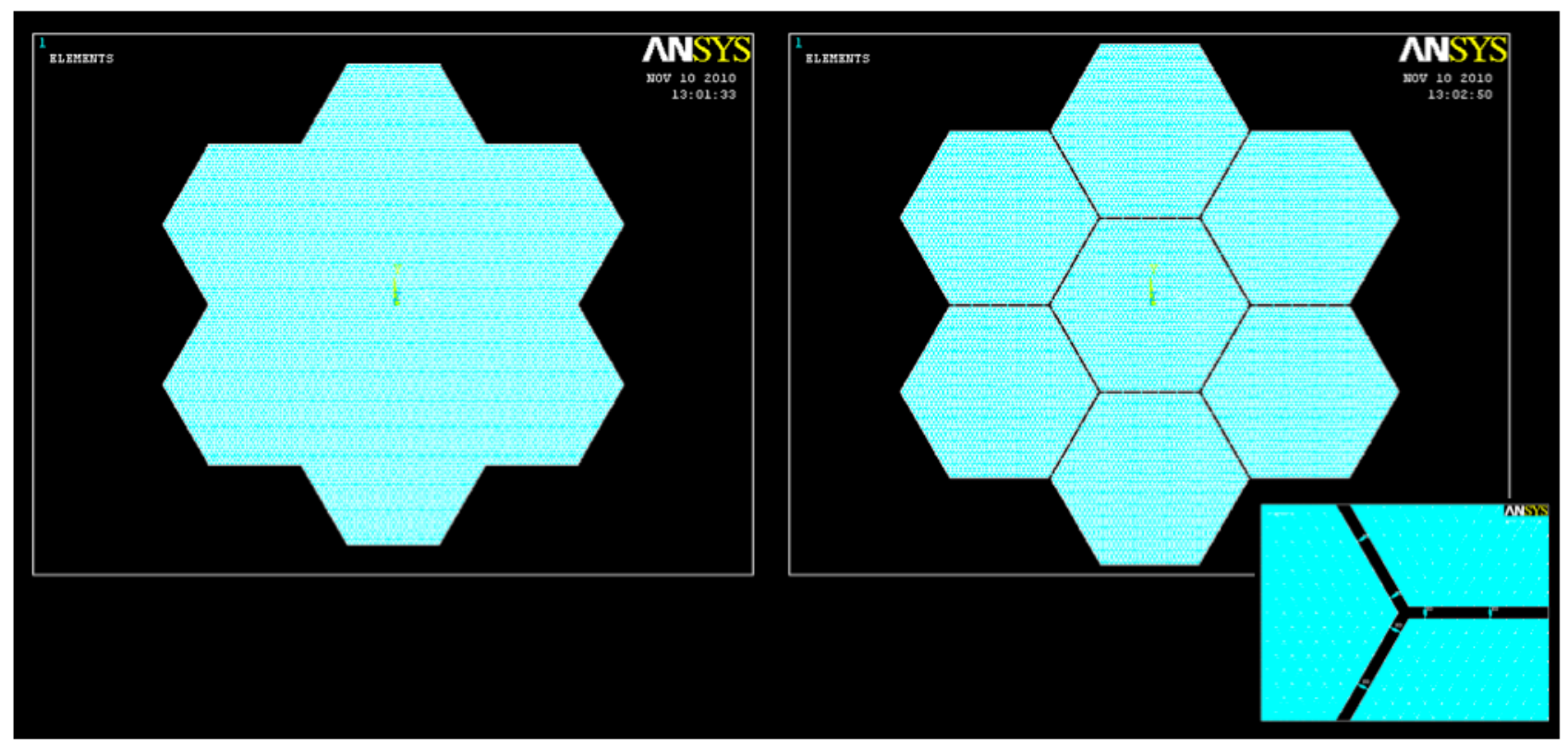

Figure 1. The ANSYS model generates a mirror using hexagonal segments, and the only difference between a segmented mirror or its equivalent monolith is the segment connectivity. For a monolith, as shown on the left, there is no gap between the segments, and the edges of adjacent segments are connected continuously. For a segmented mirror, as shown on the right, the edges of adjacent segments are connected at discrete locations by sets of damped spring elements. The inset offers a closer view of the connections between three adjacent segments.

For a monolithic mirror, there is no gap between the segments, and the edges of adjacent segments are connected continuously by merging the nodes. For a segmented mirror, the edges of adjacent segments are connected at discrete locations that correspond to the mechanism locations.

As an example, this paper considers the case of a segmented mirror whose segments are connected edgewise by flux-pinning mechanisms. ${ }^{11}$ The mechanism model assumes that all of the important dynamics can be captured by a set of four collocated single-degree-of-freedom damped spring elements. Since flux pinning is twice as stiff for motions perpendicular to the magnet-superconductor interface as it is for motions parallel to the interface, ${ }^{12,13}$ three of the damped springs correspond to translations parallel and perpendicular to the segment edges, with the spring constant for perpendicular translation, $k_{\perp}$, set to twice the spring constant for parallel translation, $k_{\|}$. The coordinate axes for each set of spring elements are also chosen to correspond to the directions parallel and perpendicular to the segment edges, as shown in Figure 2. 


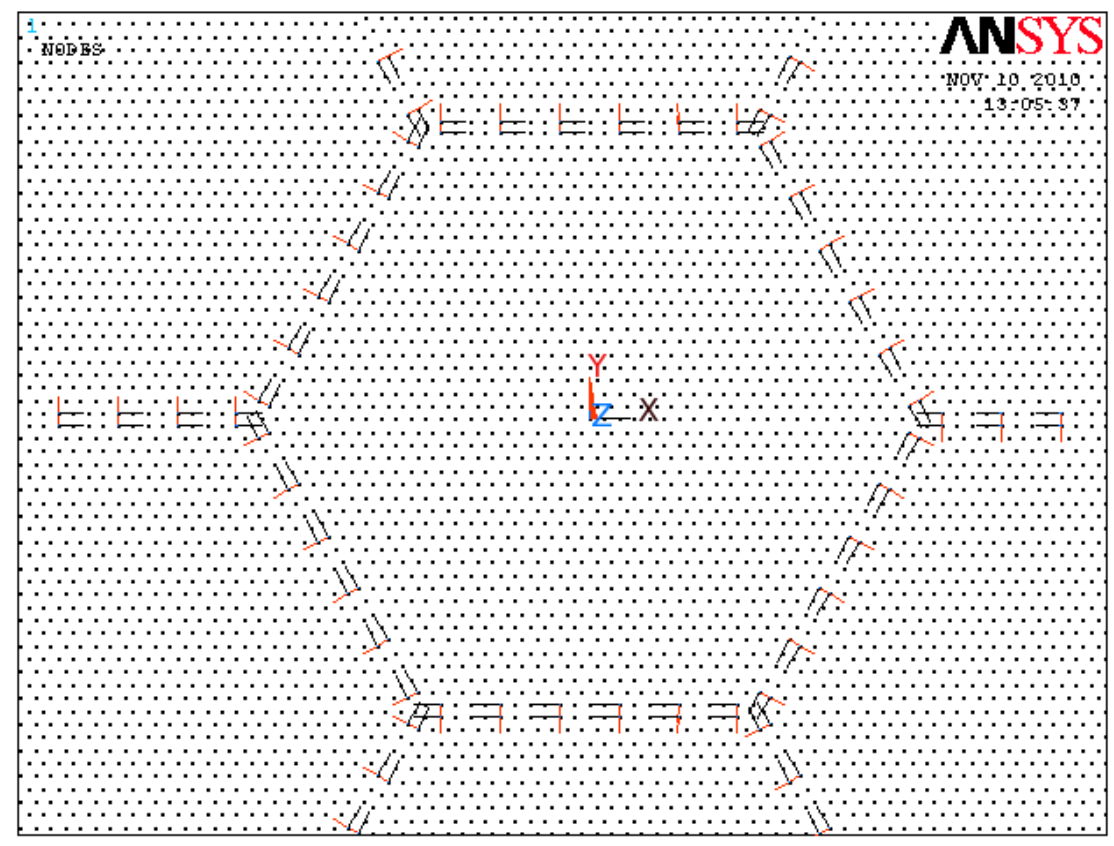

Figure 2. The flux-pinning mechanisms are represented by sets of four single-degree-of-freedom damped spring elements that correspond to translations parallel to the segment edges, translations perpendicular to the segment edges, and bending. For each set of spring elements, the nodal coordinates are rotated such that the $\mathrm{x}$-axis is parallel to the segment edge and the $\mathrm{y}$-axis is perpendicular to the segment edge.

The fourth damped spring corresponds to bending. It is assumed that the rotational spring constants for an individual mechanism are negligible, and the bending spring constant, $k_{\theta_{x}}$, is approximated using $k_{\perp}$ and the mechanism placement. Currently, the bending spring contains an implicit substructure model: for each segment edge, there are two parallel rows of mechanisms separated by a distance $d / 2$, with one row along the $x$-axis as shown in Figure 3 .

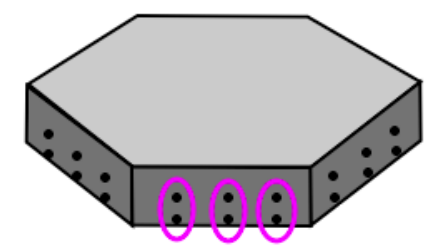

Figure 3. Since the mechanism model assumes that the rotational spring constants for an individual mechanism are negligible, multiple rows of mechanisms are required to produce the bending stiffness. In this example, there are two rows of mechanisms along the edges of each segment. The circled mechanism pairs are used to determine the bending stiffness for the corresponding spring element.

For this substructure model, the bending spring constant is approximately

$$
k_{\theta_{x}}=\frac{1}{2} k_{\perp} d,
$$

assuming small motions. For consistency, $k_{\perp}$ and $k_{\|}$are doubled to account for the two rows of springs. Finally, the case of infinitely stiff springs is considered by using the CERIG command ${ }^{14}$ for each pair of nodes that define a set of spring elements. A sample segmented mirror is shown in Figure 4. 


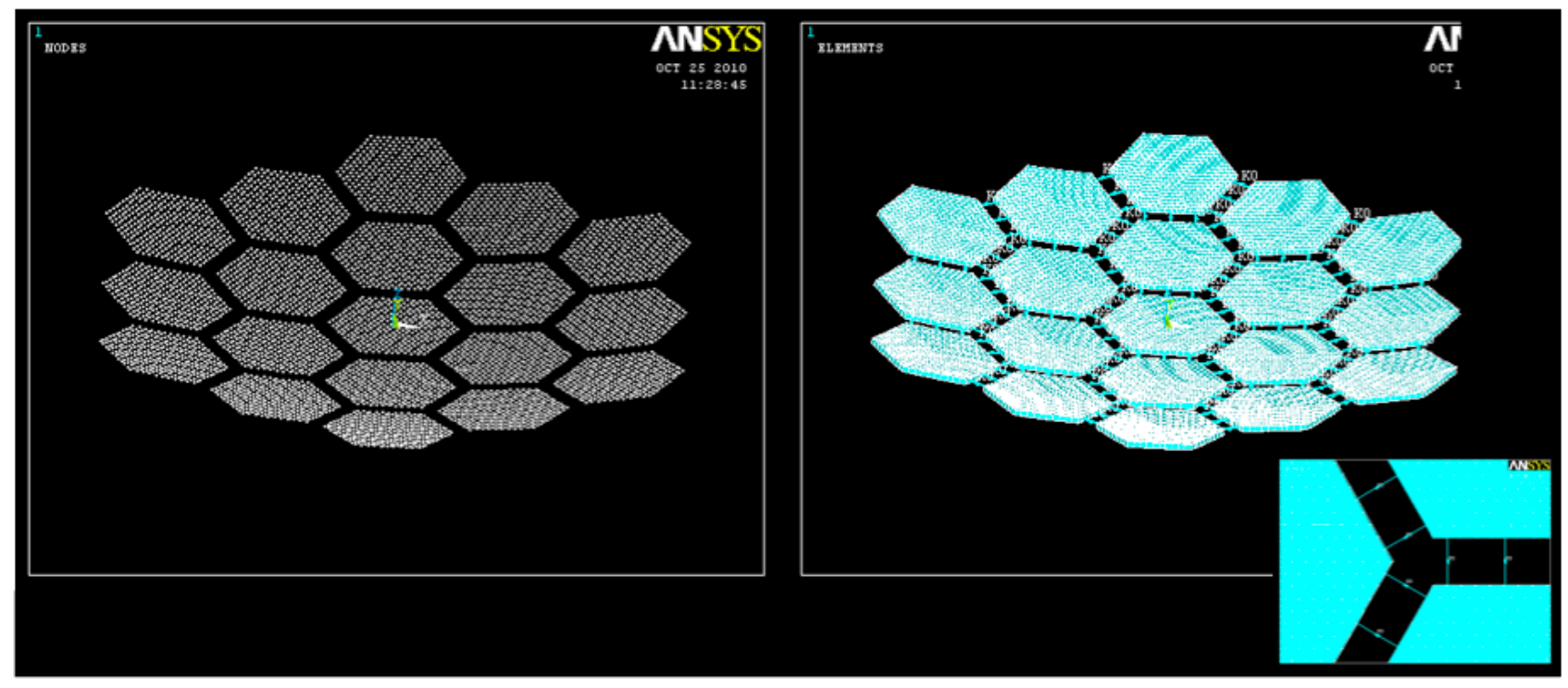

Figure 4. The figure on the left shows the nodes for a sample segmented mirror, and the figure on the right shows the corresponding elements. The inset offers a closer view of the sets of damped spring elements that model the flux-pinning mechanisms; since the four damped spring elements in each set are collocated, they appear as a single element in the inset.

\subsection{Mirror supports}

While the problem of segmented mirror mount design is beyond the scope of this paper, the mirror must be mounted in order to evaluate its disturbance response. Currently, the ANSYS model contains two mirror support options: the entire mirror is either kinematically mounted at three edge nodes spaced by $120^{\circ}$, as shown in Figure 5 , or entirely unsupported.

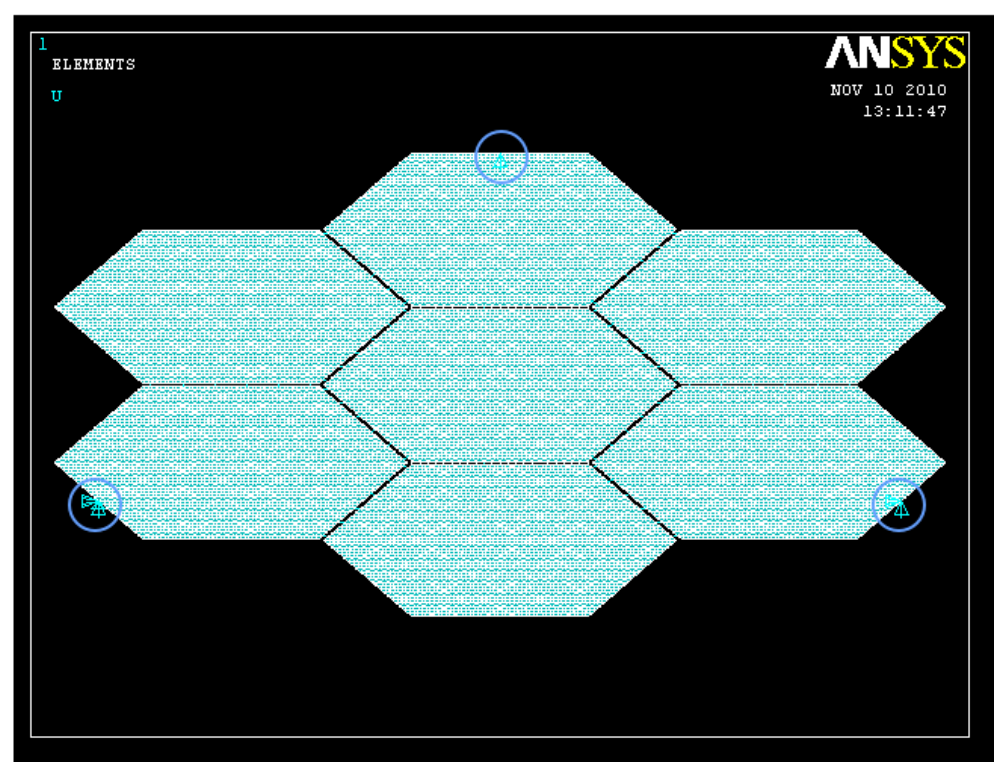

Figure 5. The kinematic support option in the ANSYS model places constraints at three edge nodes spaced by $120^{\circ}$. One of these nodes is constrained in one degree of freedom, while the other nodes are constrained in two and three degrees of freedom, respectively.

These options are sufficient for comparing the behavior of a mirror with edgewise-connected segments to the behavior of its equivalent monolith. Additional support options will be added in the future as needs arise. 


\section{PARAMETER STUDIES}

Using the ANSYS model, a series of parameter studies was run to evaluate the effects of the properties and discrete nature of the flux-pinning mechanisms on the mode shapes, natural frequencies, and disturbance response of a segmented mirror. The specific studies and their results are given below.

\subsection{Continuous vs. discrete connectivity}

When mirror segments are connected edgewise with discrete mechanisms, such as the proposed flux-pinning mechanism, the properties of the mechanism alone do not completely determine the mode shapes and natural frequencies; replacing the continuous connectivity of a monolithic mirror with discrete connections also has an effect. To separate this effect from the effects of the mechanism, the mechanism was made infinitely stiff using the CERIG command. ${ }^{14}$ Then, the mode shapes and natural frequencies were calculated. The mode shapes for a sample segmented mirror and its equivalent monolith are shown in Figure 6, and the examination of the natural frequencies is deferred until Sections 3.2 and 4.1 .

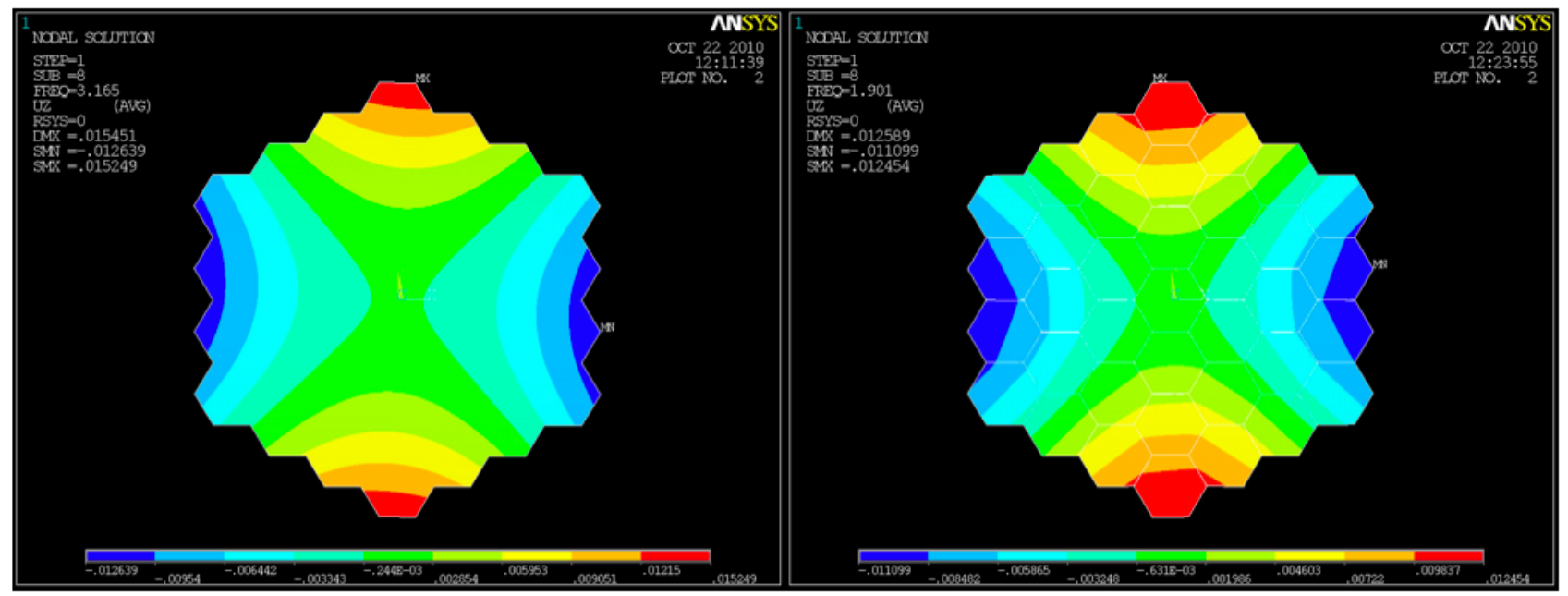

Figure 6. The figure on the left shows the first mode shape for a monolithic mirror, while the figure on the right shows the first mode shape for a segmented mirror whose segments are discretely connected edgewise. The curved contours across the monolith indicate that the entire mirror bends as a unit, while the straight lines across the segments in the segmented mirror indicate that the segments move as individual rigid bodies.

\subsection{Natural frequency design charts}

To examine how the properties of the discrete flux-pinning mechanism affect the first natural frequency, a series of parameter studies was performed. For these studies, a 15-m mirror with one ring of segments was used, with the aspect ratio varying from 80 to 120 . Along a segment edge, the number of flux-pinning mechanisms on a row varied from 3 to 8 , with the bending stiffness varying from $1 \%$ to $200 \%$ of the approximate plate constant. (The approximate plate constant, the bending stiffness of a circular plate with the same thickness and material properties as the segmented mirror, was selected as the baseline for the bending stiffness in the parameter studies since it approximated the bending stiffness of the equivalent monolith and could be calculated theoretically.) Infinitely stiff discrete connections and the equivalent monolith were also evaluated. The results of these studies are shown in Figure 7. 

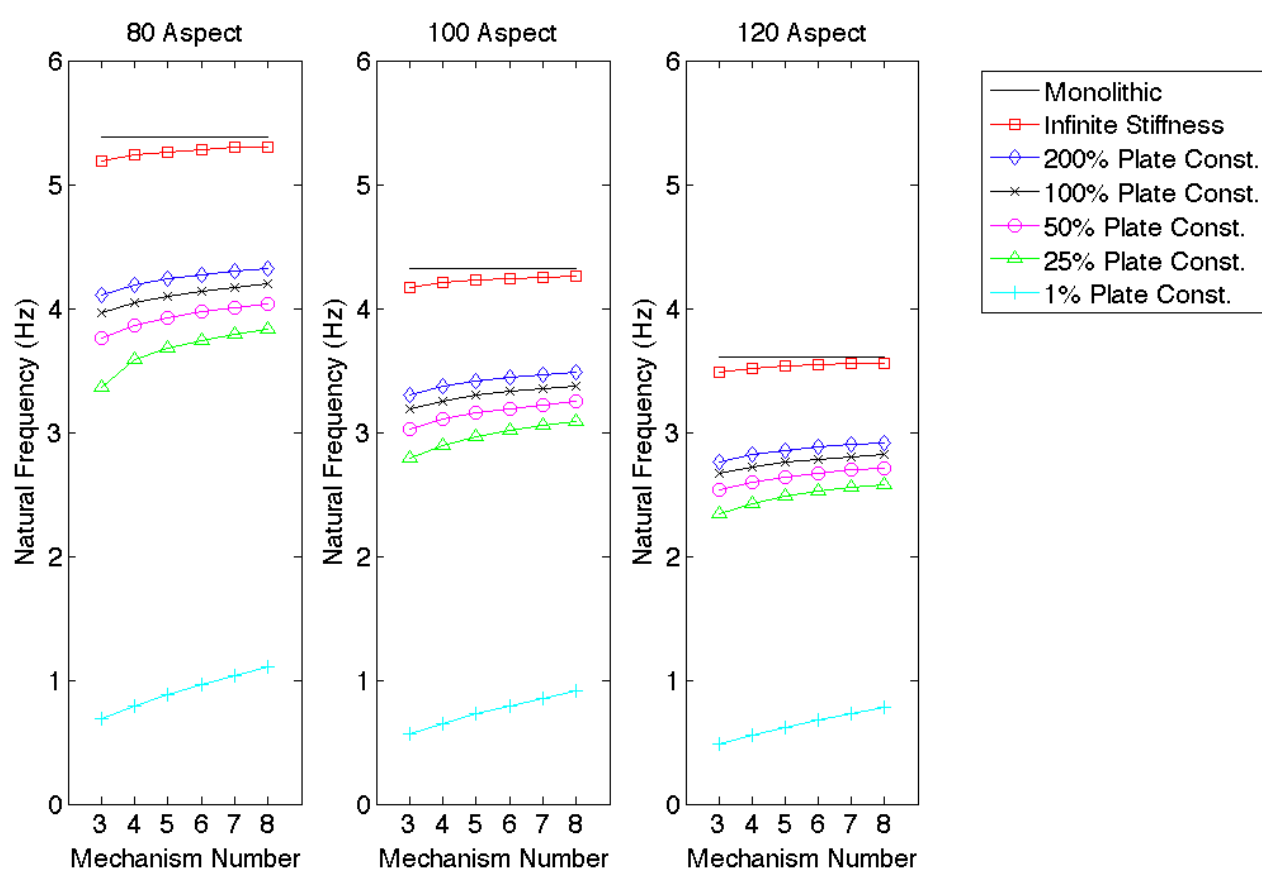

Figure 7. The first natural frequency for a segmented mirror and its equivalent monolith are shown for aspect ratios of 80 , 100, and 120 .

\subsection{The addition of discrete damping}

Finally, to investigate whether the discrete damping could affect the disturbance response, a full transient dynamic analysis was performed. The disturbance was modeled as an impulse to one of the support nodes, and the mirror response was evaluated using the root-mean-square (RMS) wavefront error, which measures how much of the mirror surface is perturbed at any given time. The time history for a sample segmented mirror is shown in Figure 8.

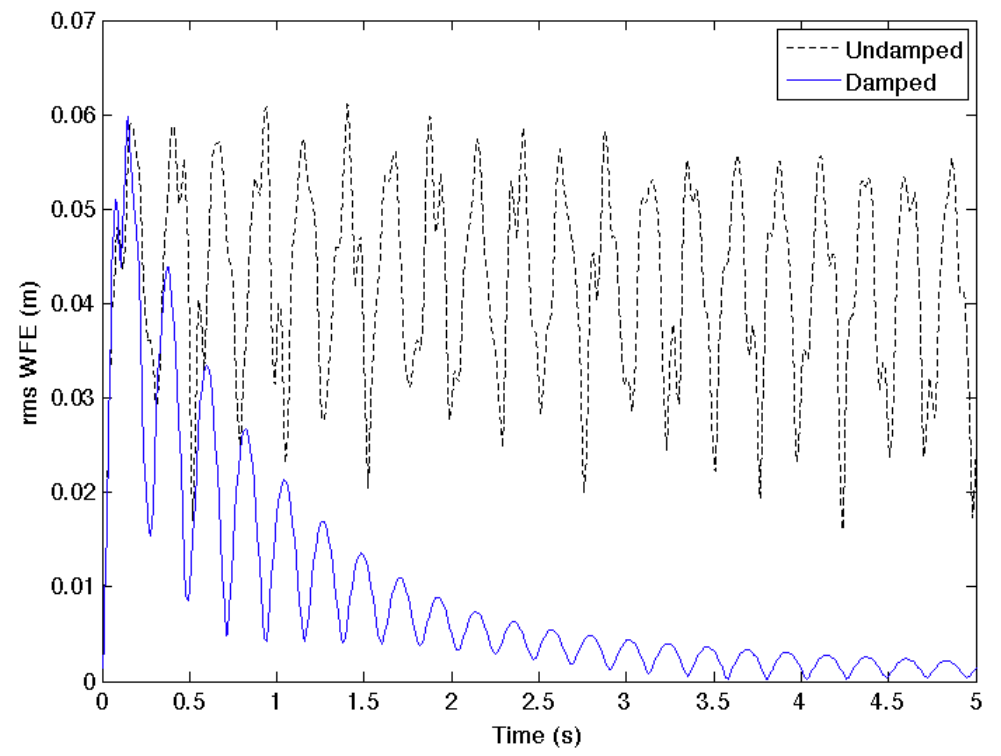

Figure 8. The disturbance response for a sample segmented mirror is shown. From this plot, it is clear that the discrete damping of the flux-pinning mechanisms can significantly improve the disturbance response, reducing the number of vibrating frequencies and damping out the vibrations in a reasonable amount of time. 


\section{DISCUSSION}

\subsection{The effect of segmentation on the mode shapes}

As shown in Figure 6, the act of replacing the continuous connectivity of a monolithic mirror with discrete edge connections does affect the mode shapes. For the monolithic mirror, the contours are curved, indicating that the mirror bends as a whole. For the segmented mirror, however, the contours are straight across the segments, indicating that the segments move as rigid bodies rather than bending. This effect is to be expected, since the discretely connected edges are less stiff than continuously connected edges, even though the individual connections are infinitely stiff. As the number of mechanisms increases, so does the amount of connectivity, and in the limit that the number of mechanisms goes to infinity, the discrete connectivity of the segmented mirror approaches the continuous connectivity of the monolithic mirror. As a result, the mode shapes of the segmented mirror approach those of the monolithic mirror as the number of mechanisms increases.

\subsection{Mechanism properties and the natural frequency}

The design curves in Figure 7 illustrate a number of trends. First, the infinite stiffness curves show that simply replacing the continuously connected edges of the monolith with discretely connected edges lowers the natural frequency. This effect is to be expected, since the discretely connected edges are less stiff than the continuously connected edges. As the number of mechanisms increases, the connectivity approaches that of a monolith, and the natural frequency asymptotes to the monolithic frequency.

For each of the finite stiffness cases, increasing the number of mechanisms increases the total spring constant and the connectivity, both of which increase the edge stiffness. As a result, the natural frequency increases. Similarly, for a fixed number of mechanisms, increasing the mechanism spring constant increases the total spring constant, leading to a higher natural frequency. Since this is true for any mechanism number, the curves never intersect. However, the curves do cluster as mechanism number or spring constant increases; since the natural frequency is proportional to the square root of the stiffness, the effect of increasing the spring constant diminishes, and as the number of connections increases, the connectivity approaches that of a monolith.

From a mechanism design standpoint, the clustering of the $25 \%, 50 \%, 100 \%$, and $200 \%$ curves is particularly interesting. When at least three mechanisms are used, the natural frequencies are all within $10 \%$ of the frequency for the $100 \%$ stiffness case. This clustering suggests that aggressively increasing the bending stiffness may not be necessary or worthwhile, especially if significant cost is involved.

\subsection{The effect of discrete damping}

From Figure 8, it is clear that the discrete damping inherent in a flux-pinning mechanism can produce a noticeable improvement in the disturbance response of a segmented mirror. In this example, the number of vibrating frequencies is reduced within the first half-second, and the mirror settles within a reasonable amount of time. However, since these results were simulated with an arbitrarily chosen damping, measurements would be needed in order to simulate the response of an actual mirror.

\section{CONCLUSIONS}

To examine the feasibility of using mechanisms analogous to damped springs to assemble the segments of a largeaperture primary mirror, the parametric ANSYS model presented in this paper has been used to compare the mode shapes, natural frequencies, and disturbance response of a mirror with flux-pinned segments to those of the equivalent monolithic mirror. Based on these parameter studies, it seems plausible that the flux-pinning mechanisms can create a segmented mirror with similar behavior as the monolith. However, fully investigating the effectiveness of a flux-pinning mechanism requires substituting actual stiffness and damping measurements into the model. The measurements vary depending upon the specific magnets and superconductors being used, and while there are numerous papers that present

measurements for magnet-superconductor separations between $1 \mathrm{~mm}$ and $10 \mathrm{~cm},{ }^{12,13,15}$ few present measurements for separations of less than $1 \mathrm{~mm}$, which more closely resembles the expected gap size between mirror segments. As a result, obtaining these measurements will be the subject of a future investigation. 
While flux-pinning mechanisms may provide a viable means of assembling mirror segments, another intriguing possibility is the use of these mechanisms as a non-contact, cryogenic damper. Traditionally, it is challenging to add damping to a cryogenic space telescope; at such low temperatures, the material damping is negligible, and other common

damping methods break down. ${ }^{16,17}$ A flux-pinning mechanism, on the other hand, requires cryogenic temperatures to operate, and it has the additional benefit of being a non-contact mechanism. The amount of damping can also be adjusted by placing nonmagnetic conductive metals, such as aluminum, near the magnet-superconductor interface. ${ }^{15}$ To examine this application, damping measurements are required, which will be the subject of a future paper.

\section{ACKNOWLEDGEMENT}

We would like to thank the NASA Graduate Student Researchers Program for its support of this project.

\section{REFERENCES}

[1] Leisawitz, D., “NASA's far-IR/submillimeter roadmap missions: SAFIR and SPECS," Advances in Space Research 34 (3), 631-636 (2004).

[2] Thronson, H. A., Postman, M., Stahl, H. P., and Traub, W., “Astronomy enabled by Ares $V$ heavy lift,” Future InSpace Operations White Paper (2007).

[3] Benford, D. J., Amato, M. J., Mather, J. C., Moseley Jr., S. H., and Leisawitz, D. T., "Mission concept for the single aperture far-infrared (SAFIR) observatory,” Astrophysics and Space Science 294, 177-212 (2004).

[4] Rieke, G. H., Benford, D. J., Harvey, P. M., Lawrence, C. R., Leisawitz, D. T., Lester, D. F., Mather, J. C., Stacey, G. J., Werner, M. W., and Yorke, H. W., "Charting the winds that change the universe, II: The single aperture farinfrared observatory (SAFIR)," Proceedings of the Second Workshop on New Concepts for Far-Infrared and Submillimeter Space Astronomy, 157-166 (2002).

[5] Mountain, M., van der Marel, R., Soummer, R., Koekemoer, A., Ferguson, H., Postman, M., Gavel, D. T., Guyon, O., Simons, D., and Traub, W. A., "Comparison of optical observational capabilities for the coming decades: ground versus space,” Astro2010: The Astronomy and Astrophysics Decadal Survey, Technology Development Papers, no. 12, (2009).

[6] Gardner, J. P., Mather, J. C., Clampin, M., Doyon, R., Greenhouse, M. A., Hammel, H. B., Hutchings, J. B., Jakobsen, P., Lilly, S. J., Long, K. S., Lunine, J. I., McCaughrean, M. J., Mountain, M., Nella, J., Rieke, G. H., Rieke, M. J., Rix, H.-W., Smith, E. P., Sonneborn, G., Stiavelli, M., Stockman, H. S., Windhorst, R. A., and Wright, G. S., “The James Webb Space Telescope,” Space Science Reviews 123 (4), 485-606 (2006).

[7] Lawson, P R., Lay, O. P., Johnston, K. J., Beichmann, C. A., Unwin, S. C., Martin, S. R., Hunyadi, S. L., Absil, O., Akeson, R. L., Bally, J., Bordé, P., Crisp, D., Danchi, W. C., Defrère, D., Eiroa, C., Falkowski, P., Henning, T., Hinz, P. M., Hollis, J. M., Hyland, D. C., Kaltenegger, L., Labadie, L., Lane, B. F., Laughlin, G., Léger, A., Liseau, R., Mawet, D., Mennesson, B., Monnier, J. D., Quillen, A. C., Röttgering, H. J. A., Selsis, F., Serabyn, E., Wilner, D. J., Woolf, N. J., Traub, W. A., Gappinger, R. O., Ksendzov, A., Peters, R. D., and Scharf, D. P., "Terrestrial Planet Finder Interferometer (TPF-I) Whitepaper for the AAAC Exoplanet Task Force,” (2007).

[8] Kong, E. M. C., Kwon, D. W., Schweighart, S. A., Elias, L. M., Sedwick, R. J., and Miller, D. W., "Electromagnetic formation flight for multisatellite arrays,” Journal of Spacecraft and Rockets 41 (4), 659-666 (2004).

[9] Labeyrie, A., "Standing wave and pellicle: A possible approach to very large space telescopes," Astronomy and Astrophysics 77, L1-L2 (1979).

[10]Labeyrie, A., Guillon, M., and Fournier, J.-M., "Optics of 'laser trapped mirrors' for large telescopes and hypertelescopes in space,” Proc. SPIE 5899, 307-316 (2005).

[11] Gersh-Range, J. A., Arnold, W. R., Peck, M. A., and Stahl, H. P., “A flux-pinning mechanism for segment assembly and alignment," Proc. SPIE 8150 (to appear).

[12] Basinger, S. A., Hull, J. R., and Mulcahy, T. M., "Amplitude dependence of magnetic stiffness in bulk hightemperature superconductors,” Applied Physics Letters 57 (27), 2942-2944 (1990).

[13]Hull, J. R. and Cansiz, A., "Vertical and lateral forces between a permanent magnet and a high-temperature superconductor,” Journal of Applied Physics 86 (11), 6396-6404 (1999). 
[14] ANSYS, [ANSYS, Inc. Documentation for Release 12.0], ANSYS, Inc., Canonsburg, PA, (2009).

[15] Shoer, J. P. and Peck, M. A., "Flux-pinned interfaces for the assembly, manipulation, and reconfiguration of modular space systems,” The Journal of the Astronautical Sciences 57 (3), 667-688 (2009).

[16] Romberg, O., Tausche, M., Pereira, C., and Panning, L., "Passive damping of spacecraft sandwich panels," $10^{\text {th }}$ European Conference on Spacecraft Structures, Materials, \& Mechanical Testing, 1-8 (2007).

[17] Bely, P. Y. (ed.), [The Design and Construction of Large Optical Telescopes], Springer-Verlag, New York, 260-261 (2003). 


\section{A Parametric Finite-Element Model for Evaluating Segmented Mirrors with Discrete, Edgewise Connectivity}

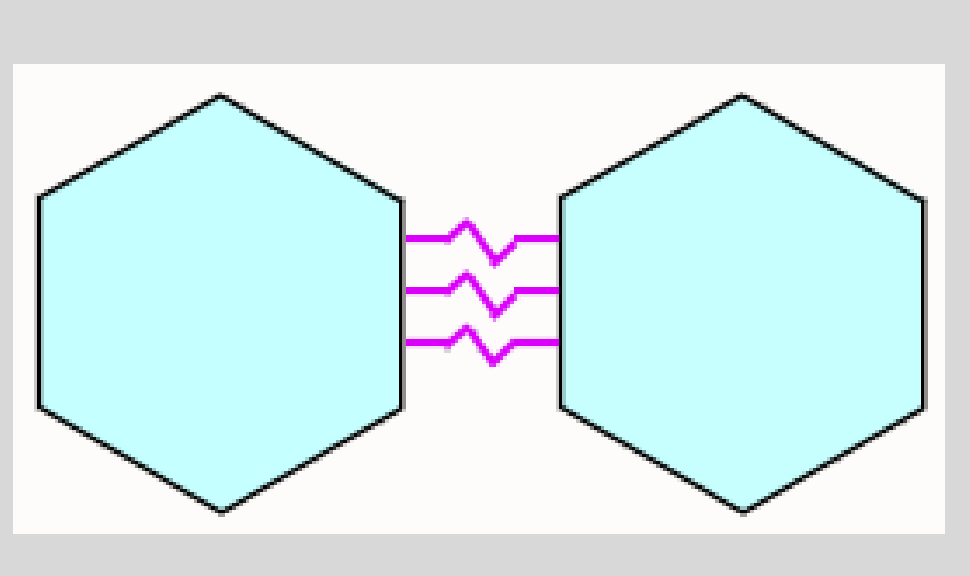

Jessica A. Gersh-Range William R. Arnold Mason A. Peck

H. Phillip Stahl

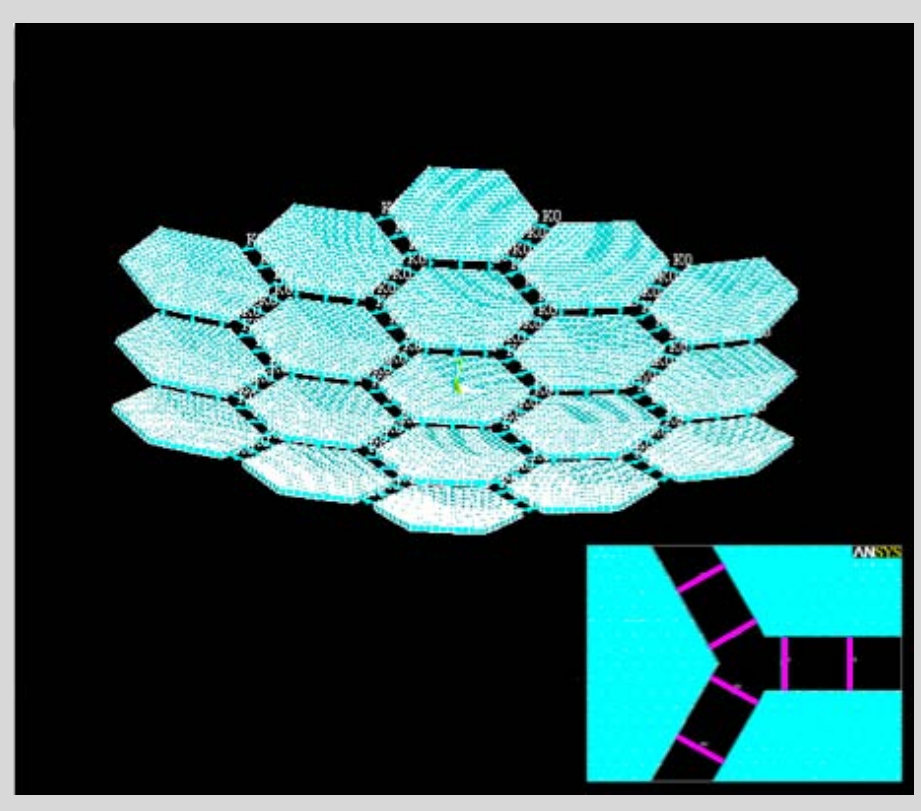




\section{Introduction}

Current astrophysics topics include:

-Early star formation

-Planetary evolution

-The search for life elsewhere

These topics create a need for space telescopes with apertures of at least 10 meters. 


\section{Introduction}

Introduction

Edgewise

Connectivity

The Model

Parameter

Studies

Conclusions
The assembly and deployment of such large space telescopes is a challenge.

Possible schemes include:

-Launching a monolith

-Scaling the design of the James Webb Space Telescope

-Assembling a collection of primary mirror segments on-orbit 


\section{Introduction}

\section{On-orbit assembly methods include:}

-Formation flight

-Electromagnetic formation flight

- Laser trapping

These methods treat the primary mirror as an array of free-floating segments and rely on active control to prevent collisions and maintain optical alignment. 


\section{Edgewise Connectivity}

Introduction

\section{Edgewise}

Connectivity

The Model

Parameter

Studies

Conclusions
-Neighboring segments connected by mechanisms analogous to damped springs.

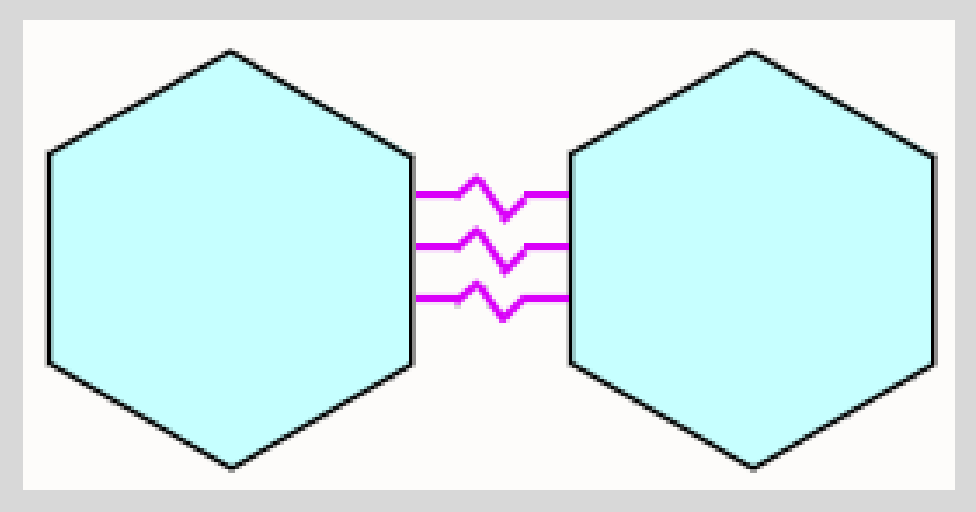

-These mechanisms maintain the alignment after the segments are connected.

- It is assumed that some other method maintains the alignment while the segments are connected.

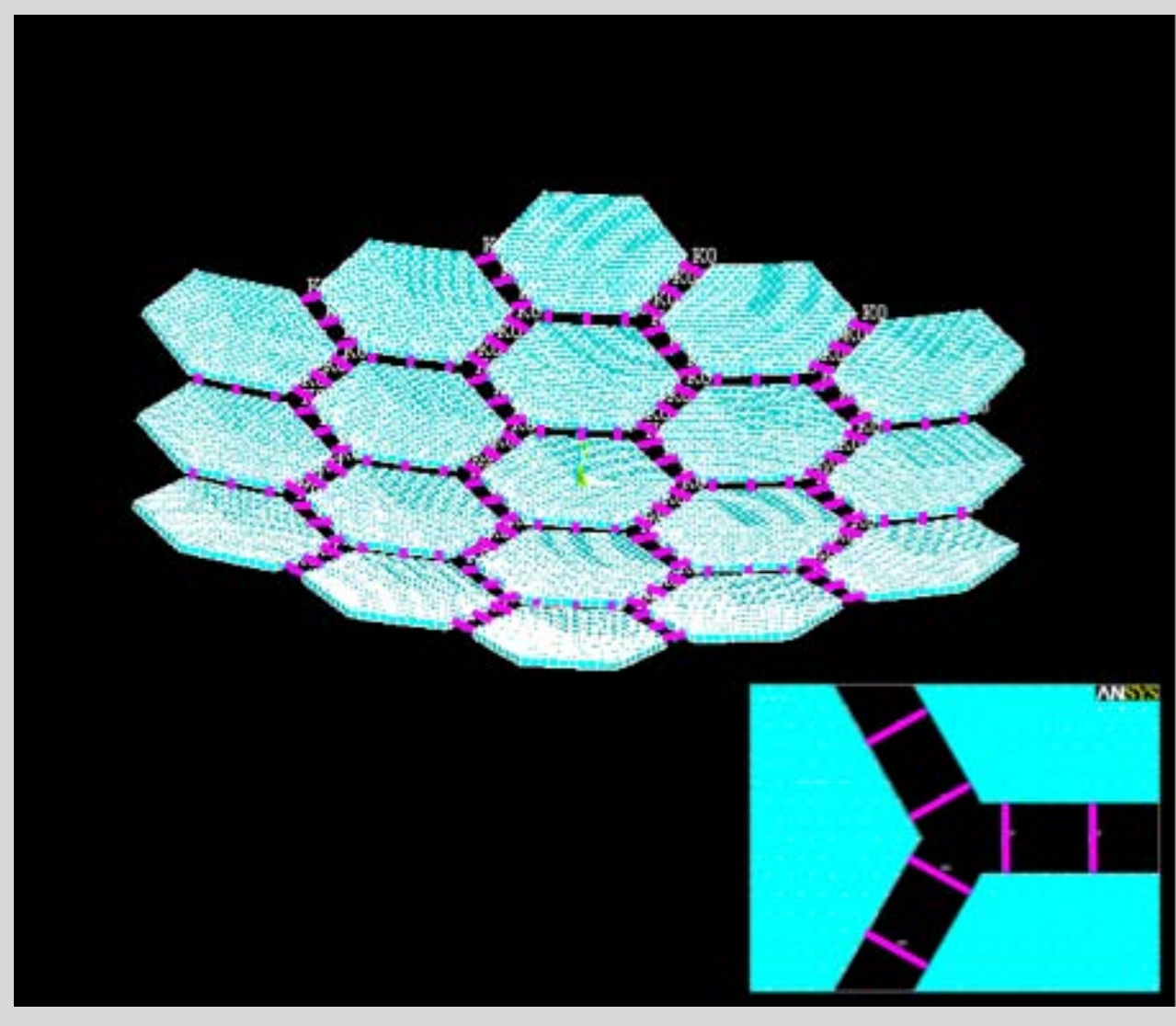




\section{The Finite-Element Model}

Introduction

\section{Edgewise}

Connectivity

The Model

Parameter

Studies

\section{Conclusions}

\section{Mirror Model Parameters:}

-Mirror diameter

-Size of the gap between segments (can be 0)

-Number of rings

-Mirror curvature (can be flat or parabolic)

-Aspect ratio

-Mirror type (segmented or monolithic) 


\section{The Finite-Element Model}

Introduction

Edgewise

Connectivity

The Model

Parameter

Studies

Conclusions
Equivalent Monolith: Edges of adjacent segments connected continuously

Segmented Mirror: Edges of adjacent segments connected at discrete locations by damped springs
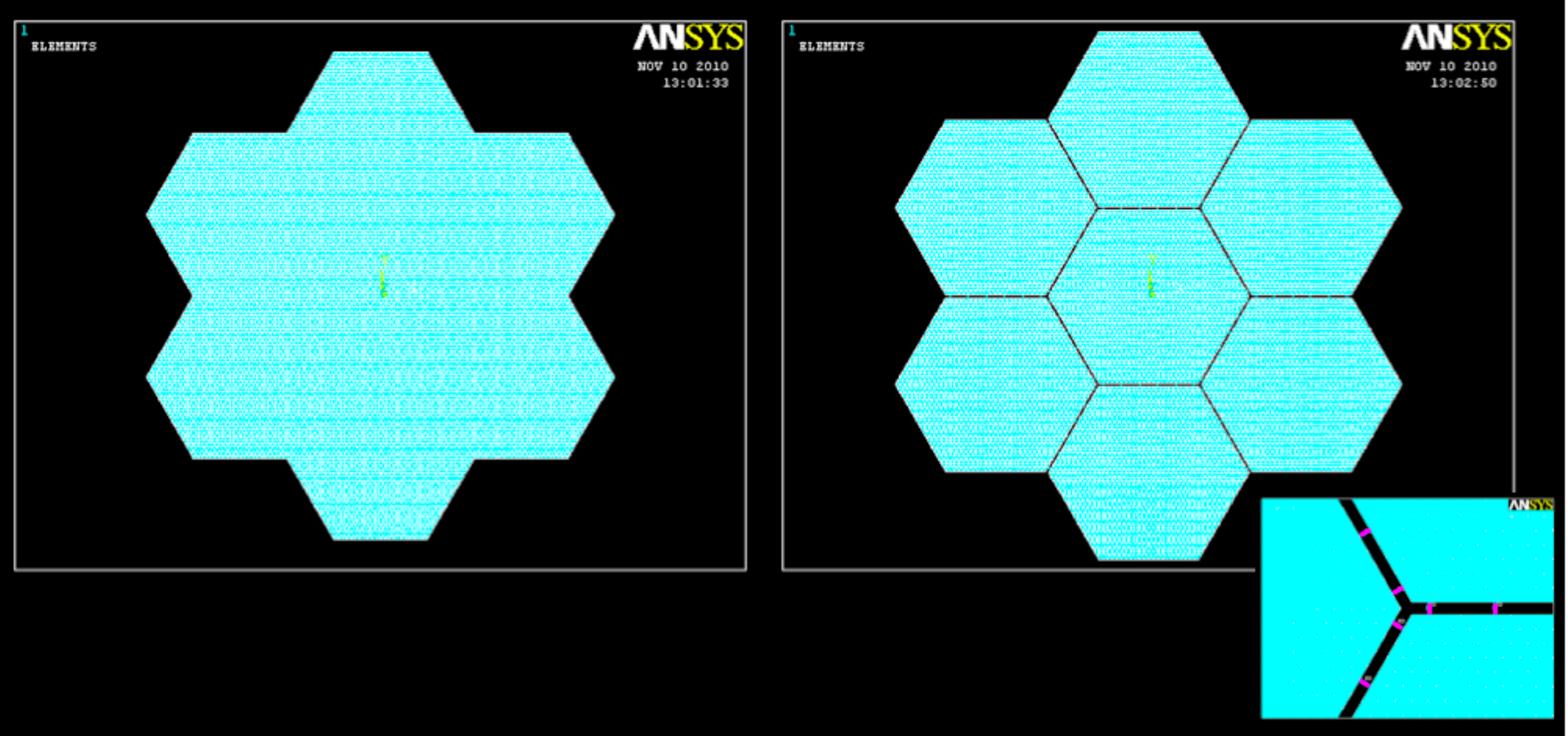


\section{Mechanism Model}

Introduction

\section{Edgewise}

\section{Connectivity}

The Model

Parameter

Studies

Conclusions

\section{Example: Flux-Pinning Mechanisms}

-Modeled as sets of four collocated single-degree-of-freedom springs.

-Three springs correspond to translations parallel and perpendicular to the segment edges.

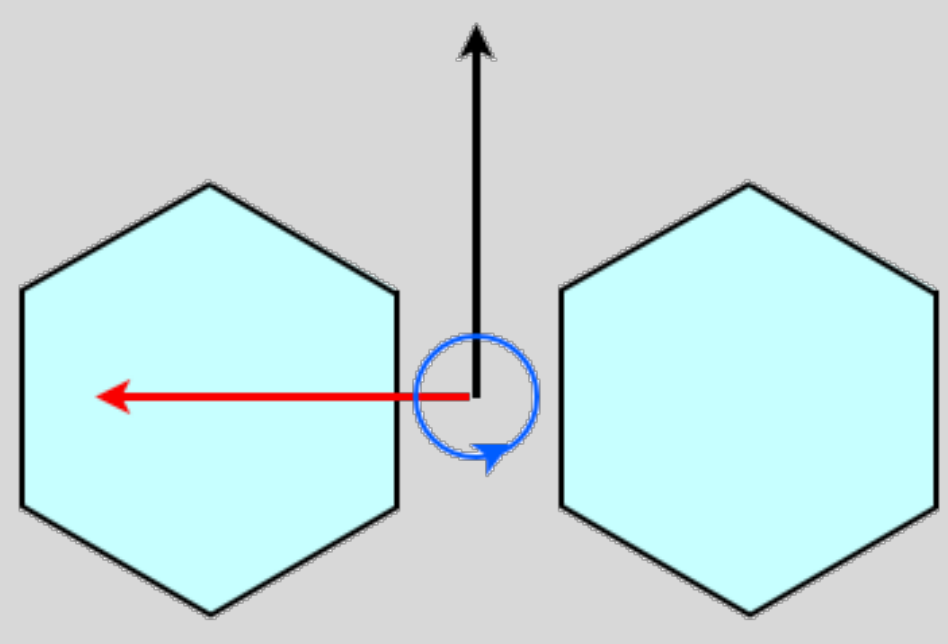

-The spring constant for perpendicular translation is twice the spring constant for parallel

$$
k_{\text {丩 }}=2 k_{\mathrm{F}}
$$




\section{Mechanism Model}

Introduction

\section{Edgewise}

Connectivity

The Model

Parameter

Studies

Conclusions
Example: Flux-Pinning Mechanisms

-The fourth spring corresponds to bending.

-Multiple rows of mechanisms are required to produce the bending stiffness.

-The stiffness is approximated using $k_{\perp}$ and the spacing between the rows:

$$
k_{\theta_{x}}=\frac{1}{2} k_{\perp} d
$$

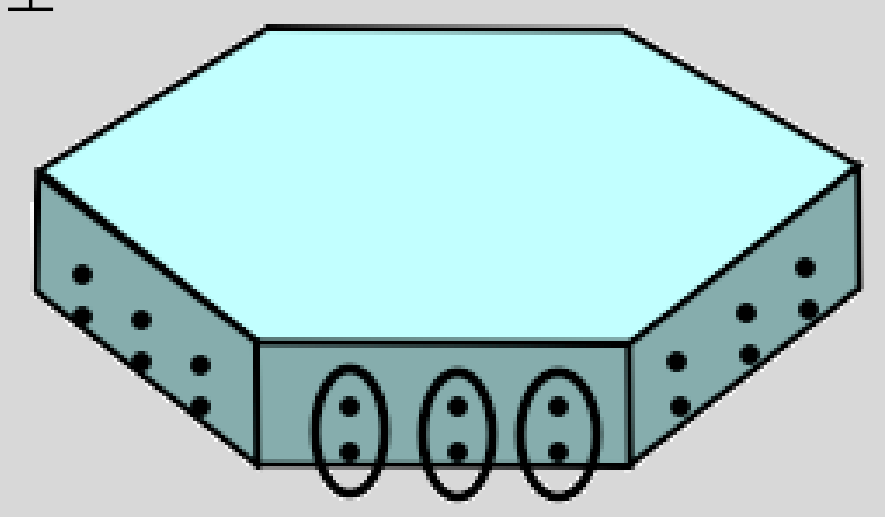




\section{Sample Segmented Mirror}

Introduction

Edgewise

Connectivity

The Model

Parameter

Studies

Conclusions
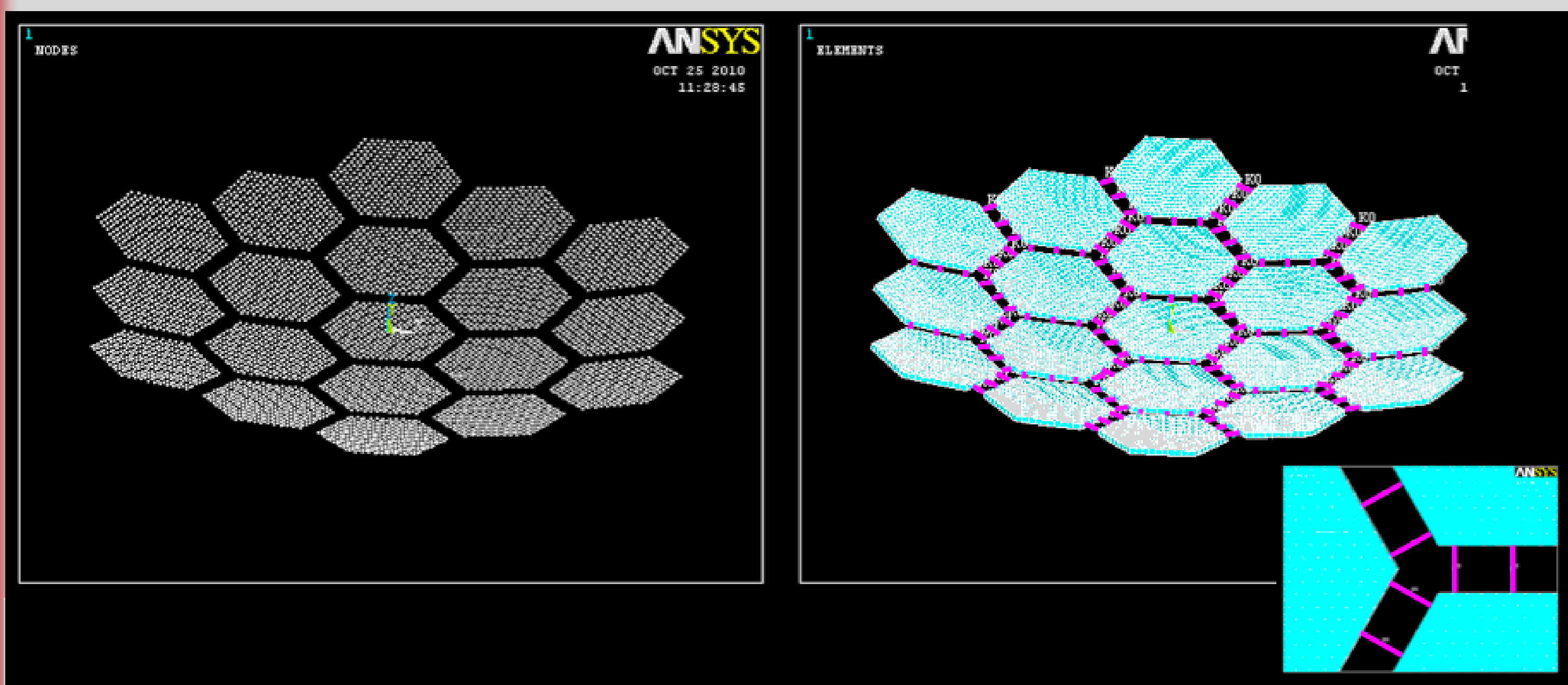


\section{Mirror Supports}

Introduction

\section{Edgewise}

Connectivity

The Model

Parameter

Studies

Conclusions

\section{Mirror Support Options:}

-Unsupported

-Kinematically mounted at three edge nodes

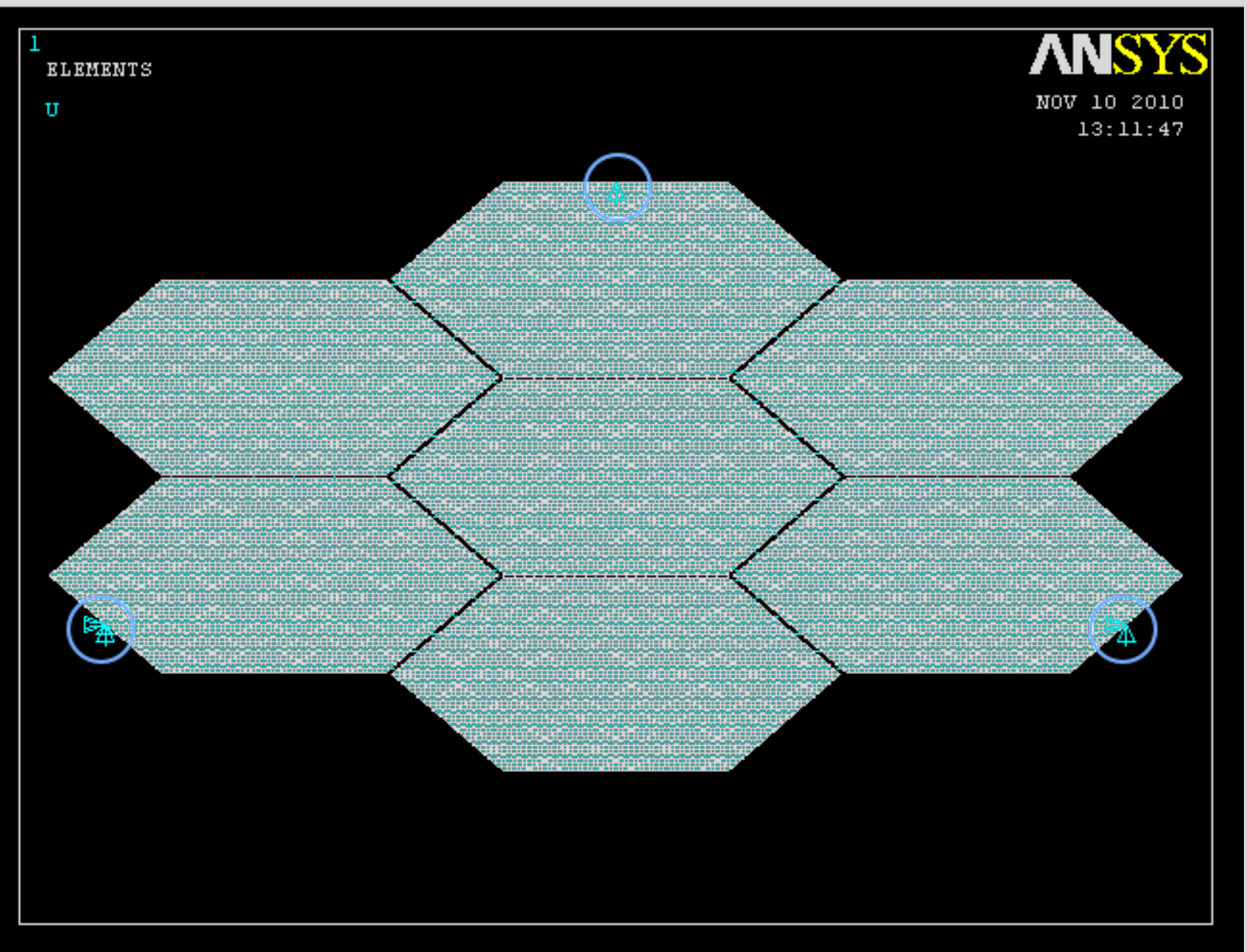




\section{Continuous vs. Discrete Continuity}

Introduction

Edgewise

Connectivity

The Model

Parameter Studies

Conclusions

Monolithic Case: Contours curved, indicating mirror bends as a unit

Segmented Case: Contours straight across the segments, indicating segments move as rigid bodies
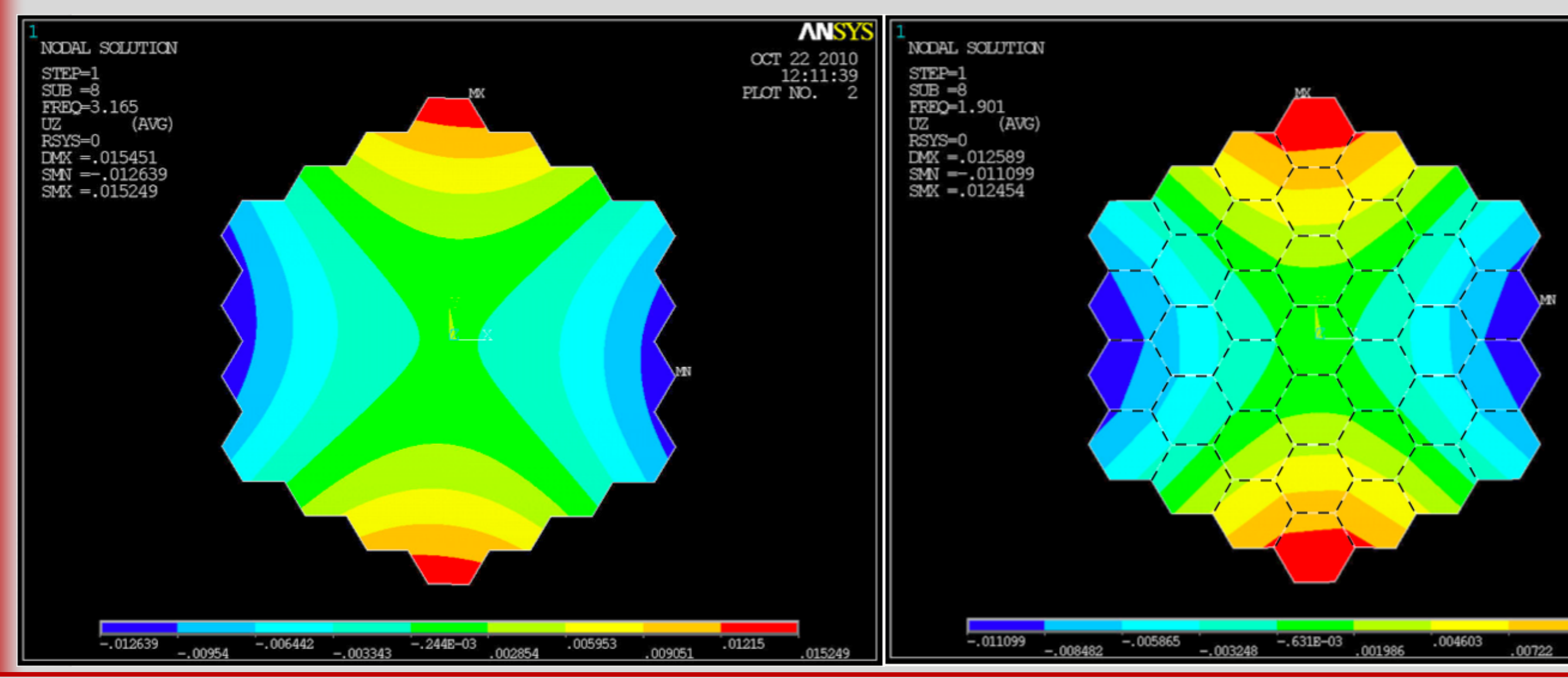

NNSY ProT $12: 23: 55$ 


\section{Natural Frequency Design Charts}

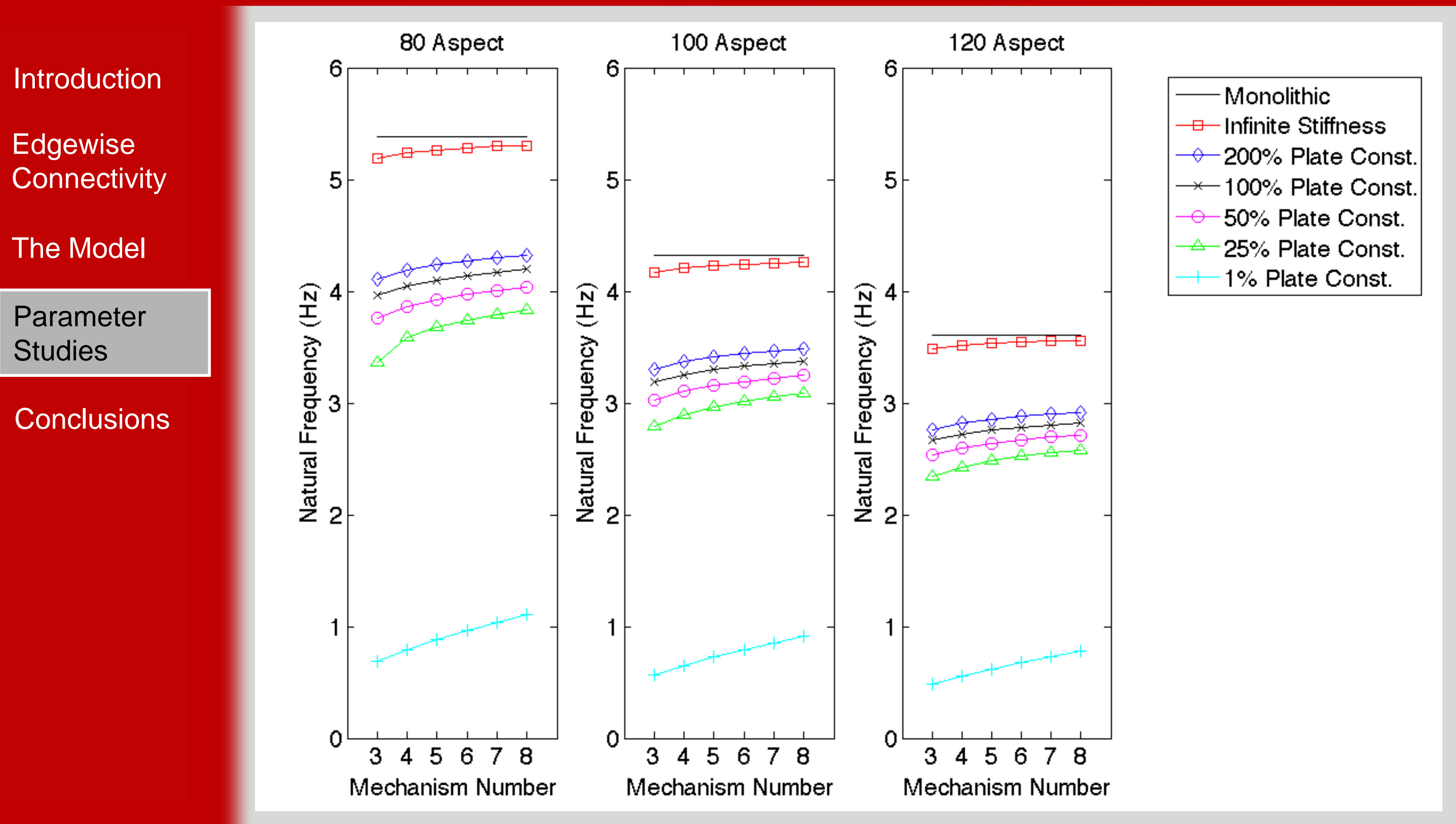




\section{The Effects of Discrete Damping}

\section{Introduction}

Edgewise

Connectivity

The Model

\section{Parameter}

Studies

\section{Conclusions}

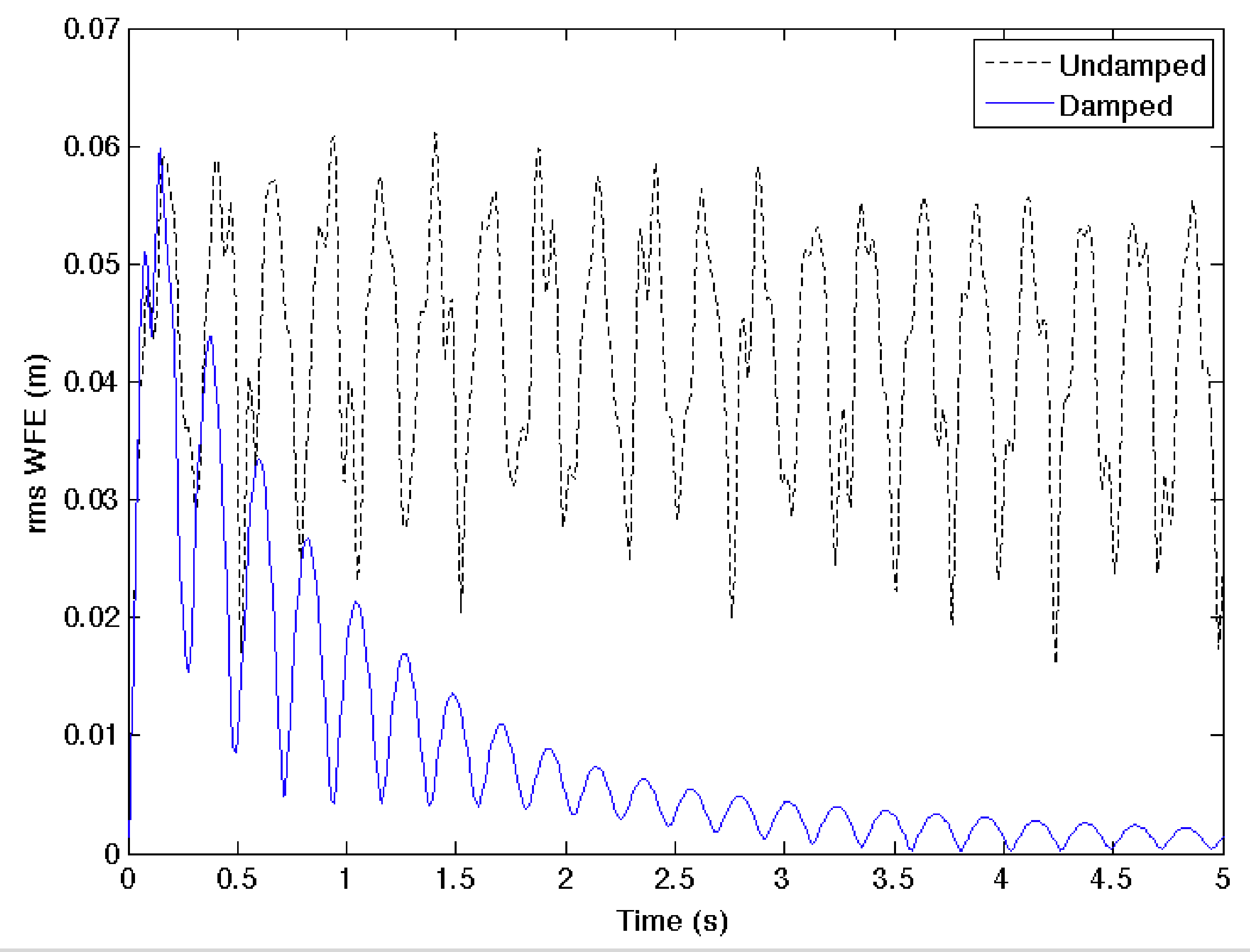




\section{Conclusions}

Introduction

Edgewise

Connectivity

The Model

Parameter

Studies

Conclusions
-By connecting the segments edgewise, it might be possible to construct a segmented mirror that behaves similarly to a monolith.

-The mirror behavior depends upon the specific mechanism parameters.

-The discrete nature of the connections also affects the behavior.

-Flux-pinning mechanisms may also be useful as non-contact, cryogenic dampers. 